\title{
Factors Associated with the Use of Different Treatment Modalities among Patients with Upper Airway Diseases in Taiwan: A Cross-Sectional Survey Study
}

\author{
Malcolm Koo, ${ }^{1,2}$ Kai-Li Liang, ${ }^{3,4,5}$ Hsin Tsao, ${ }^{6}$ Ting-Ting Yen, \\ Rong-San Jiang, ${ }^{3,4}$ and Yueh-Chiao Yeh $^{6,7}$ \\ ${ }^{1}$ Department of Medical Research, Buddhist Dalin Tzu Chi Hospital, No. 2, Minsheng Road, Dalin, Chiayi 62247, Taiwan \\ ${ }^{2}$ Dalla Lana School of Public Health, University of Toronto, No. 155, College Street, Toronto, ON, Canada M5T 3M7 \\ ${ }^{3}$ Department of Otolaryngology, Taichung Veterans General Hospital, No. 1650, Sec. 4, Taiwan Boulevard, \\ Taichung City 40705, Taiwan \\ ${ }^{4}$ School of Medicine, Chung Shan Medical University, No. 110, Sec. 1, Jianguo North Road, Taichung City 40201, Taiwan \\ ${ }^{5}$ Department of Medicine, National Yang-Ming Medical University, No. 155, Sec. 2, Linong Street, Taipei 11221, Taiwan \\ ${ }^{6}$ Graduate Institute of Natural Healing Sciences, Nanhua University, No. 55, Sec. 1, Nanhua Road, Dalin, Chiayi 62249, Taiwan \\ ${ }^{7}$ Department of Natural Biotechnology, Nanhua University, No. 55, Sec. 1, Nanhua Road, Dalin, Chiayi 62249, Taiwan
}

Correspondence should be addressed to Rong-San Jiang; rsjiang@vghtc.gov.tw and Yueh-Chiao Yeh; ycyeh@mail.nhu.edu.tw

Received 10 May 2013; Revised 6 August 2013; Accepted 8 August 2013

Academic Editor: William E. Berger

Copyright (C) 2013 Malcolm Koo et al. This is an open access article distributed under the Creative Commons Attribution License, which permits unrestricted use, distribution, and reproduction in any medium, provided the original work is properly cited.

\begin{abstract}
Rhinitis is a common upper airway disease and can have great impact on patients' quality of life. Factors associated with the use of common treatment modalities among 279 Taiwanese rhinitis patients from the outpatient department of otolaryngology in a medical center were investigated using a cross-sectional survey study. Results from multiple logistic regression analysis, adjusted for etiologies of rhinitis, revealed that males were associated with surgical intervention $(\mathrm{OR}=2.11, P=0.009)$. Lower educational level was associated with oral $(\mathrm{OR}=2.31, P=0.024)$ and topical medications $(\mathrm{OR}=2.50, P=0.005)$. Poor or fair general health status was associated with topical medications $(\mathrm{OR}=4.47, P=0.001)$, whereas very good or excellent general health status was inversely associated with surgical intervention $(\mathrm{OR}=0.32, P=0.002)$. Smoking was associated with the use of nasal irrigation $(\mathrm{OR}=2.72, P=0.003)$. Worse disease-specific quality of life was associated with oral medications $(\mathrm{OR}=2.46, P=0.010)$ and traditional Chinese medicine $(\mathrm{OR}=5.43, P<0.001)$. In conclusion, the use of different treatment modalities for rhinitis was associated with different combinations of independent factors.
\end{abstract}

\section{Introduction}

Rhinitis is the inflammation of the mucous lining of the nose, and it can lead to symptoms including rhinorrhea, nasal obstruction, post-nasal drip, itching, and sneezing. The etiologies of rhinitis include infection, anatomical anomalies, immunological disorders, hormonal imbalance, and ciliary defects $[1,2]$. Similar nasal symptoms can be caused by different etiologies, making the diagnosis and treatment of rhinitis difficult.

Methods for management of rhinitis include environmental control, pharmacotherapy, immunotherapy, surgical interventions, nasal irrigation, complementary, and alternative medicine [3]. Medications used for rhinitis are usually administered intranasally or orally. The surgical indications for rhinitis include drug-resistant inflammatory mucosal hypertrophy, anatomical anomalies, and sinus drainage obstruction. Complementary or alternative medicines such as traditional Chinese medicine (TCM), acupuncture, herbs, and probiotics are also being used for the treatment of rhinitis [4-6]. Recently, evidence-based guidelines with several algorithm-guided therapeutic schemes for the treatment of rhinitis are available $[2,7,8]$. Yet, to our knowledge, no studies have been conducted to determine the factors associated with 
the use of different treatment modalities. Therefore, the aim of this study was to investigate the factors associated with the use of treatment among patients with rhinitis in Taiwan.

\section{Methods}

2.1. Study Design and Subjects. Patients with physiciandiagnosed rhinitis from the outpatient department of otolaryngology in Taichung Veterans General Hospital, a medical center in central Taiwan, were invited to participate in this cross-sectional survey study. The diagnosis of rhinitis was based on patients' reports of typical nasal symptoms persisting for two weeks or more and rhinoscopy examination. Typical nasal symptoms include rhinorrhea, nasal obstruction, postnasal dripping, itching, and sneezing. Physical examination with anterior rhinoscopy or nasal endoscopy was performed by two rhinologists (RSJ and KLL). All enrolled patients revealed signs of nasal inflammation including mucosal edema, nasal polyp, polypoid swelling, discharge (purulent, mucous or serous), or crust. Patients under 20 years old or with sinonasal tumors were excluded from the study. The study was approved by the Institutional Review Board of Taichung Veterans General Hospital, and all participants gave written informed consent.

2.2. Data Collection. Each patient completed a questionnaire with questions on sociodemographic status, lifestyle, general health status, disease-specific quality of life, and previous use of treatment modalities for rhinitis. The treatment modalities were classified into four main categories: pharmacology, surgical intervention, TCM, and nasal irrigation. Pharmacology was further subdivided into oral medications and topical medications. The disease-specific quality of life was assessed using the Chinese version of the 31-item rhinosinusitis outcome measure (CRSOM-31) [9]. The CRSOM-31 is a validated instrument translated from the widely used rhinosinusitis outcome measure (RSOM-31) [10]. It contains seven domains including nasal symptoms (6 items), eye symptoms (3 items), sleep (3 items), ear symptoms (5 items), general symptoms (7 items), practical problems (4 items), and emotional consequences (3 items) for evaluation of the rhinitis or rhinosinusitis-related impact on the quality of life. For each symptom, there are two response scales: magnitude ( 0 to 5 ) and importance ( 1 to 4 ). The CRSOM-31 symptomimpact score is the product of the magnitude and importance scores, with higher scores indicating worse disease-specific quality of life. Etiologies of rhinitis were ascertained through medical records.

2.3. Statistical Analysis. Continuous data were expressed as mean \pm standard deviation (SD), and categorical data were expressed as frequencies and percentages. Univariate logistic regression analyses were conducted to assess the odds ratios and $95 \%$ confidence intervals for each of the treatment modalities with the independent variables, including sex, age, body mass index (BMI), marital status, educational level, alcohol use, smoking, regular exercise, general health status, CRSOM-31 symptom-impact scores, and four etiologies of rhinitis. Multivariate logistic regression analyses with backward stepwise selection method were used to evaluate the independent factors associated with the use of each of the treatment modalities. In all regression analyses, age was categorized into five groups. BMI was calculated as weight (in kilograms) divided by height (in meters) squared. Based on the definition from the Bureau of Health Promotion, Department of Health, Taiwan, the respondents were categorized as underweight (BMI $\left.<18.5 \mathrm{~kg} / \mathrm{m}^{2}\right)$, normal weight (BMI 18.5-23.9 kg/m²), overweight (BMI 24.0-26.9 kg/m²), or obese $\left(B M I \geq 27.0 \mathrm{~kg} / \mathrm{m}^{2}\right)$. Educational levels were divided into elementary school or lower (grade 1 to grade 6) and high school or above. General health status of the patients was grouped into three levels (poor or fair, good, and very good or excellent). CRSOM-31 symptom-impact scores were divided into tertiles. All computations were performed using SPSS version 17.0 (SPSS, Inc., Chicago, IL, USA). Two-tailed $P$ values $<0.05$ were considered statistically significant.

\section{Results}

A total of 279 patients with acute or chronic rhinosinusitis, allergic or nonallergic rhinitis were successfully interviewed between July and September 2011. The mean age of the patients was 48.6 years and $58.4 \%$ were males. The basic characteristics of the study participants are summarized in Table 1.

In terms of the use of treatment modalities, $85.7 \%$ of the patients had used pharmacology $(77.1 \%$ had used oral medications and $65.9 \%$ had used topical medications), 38.4\% had used surgical intervention, 34.4\% had used TCM, and $34.1 \%$ had used nasal irrigation (Table 2). In addition, 19 $(6.8 \%)$ patients had not used any treatment modalities, and $56(20.1 \%), 63(22.6 \%), 77$ (27.6\%), 36 (12.9\%), and $28(10.0 \%)$ patients had used $1,2,3,4$, and 5 treatment modalities, respectively. Regarding the severity of the rhinitis, since only 56 patients $(20 \%)$ used only a single treatment modality, there are too few individuals to provide statistically meaningful comparisons between the five treatment modalities. Nonetheless, when the total number of modalities ( 0 to 5) used by the patients was compared, we found that the CRSOM-31 scores in patients who had used all five modalities $(269.4 \pm 130.3)$ significantly higher compared to those who had used fewer modalities. No significant differences in CRSOM-31 scores were observed among those patients who had used $4(196.6 \pm 107.1), 3(173.7 \pm 85.8), 2(162.6 \pm 93.3)$, $1(148.3 \pm 101.4)$, or no $(125.7 \pm 88.7)$ treatment modalities.

Table 2 also showed the results of univariate logistic regression analyses for each of the six treatment modalities with all the independent variables. Furthermore, six separate multivariate logistic regression analyses were conducted to assess the association between each of the treatment modalities and their independent and significant factors (Table 3). Male sex was significantly associated with the use of surgical intervention. Educational levels of elementary school or below were significantly associated with the use of pharmacology treatment modality and also with the use of subgroup of pharmacology treatment modality (oral or 
TABLE 1: Characteristics of study participants $(N=279)$.

\begin{tabular}{|c|c|}
\hline Characteristic & $n(\%)$ \\
\hline \multicolumn{2}{|l|}{ Sex } \\
\hline Female & $116(41.6)$ \\
\hline Male & $163(58.4)$ \\
\hline Age $(y r)$ & $48.6 \pm 18.1(50.0,20-89)^{*}$ \\
\hline$\leq 30$ & $54(19.4)$ \\
\hline $31-40$ & $46(16.5)$ \\
\hline $41-50$ & $47(16.8)$ \\
\hline $51-60$ & $69(24.7)$ \\
\hline$>60$ & $63(22.6)$ \\
\hline Body mass index $\left(\mathrm{kg} / \mathrm{m}^{2}\right)$ & $24.0 \pm 3.8(23.8,12.3-35.9)^{*}$ \\
\hline Underweight & $12(4.3)$ \\
\hline Normal & $141(50.5)$ \\
\hline Overweight & $72(25.8)$ \\
\hline Obese & $54(19.4)$ \\
\hline \multicolumn{2}{|l|}{ Marital status } \\
\hline Single, divorced, widowed, or other & $94(33.7)$ \\
\hline Married & $185(66.3)$ \\
\hline \multicolumn{2}{|l|}{ Educational level } \\
\hline Elementary school or below & $78(28.0)$ \\
\hline High school or above & $201(72.0)$ \\
\hline \multicolumn{2}{|l|}{ General health status } \\
\hline Poor or fair & $55(19.7)$ \\
\hline Good & $158(56.6)$ \\
\hline Very good or excellent & $66(23.7)$ \\
\hline \multicolumn{2}{|l|}{ Smoking } \\
\hline No & $232(83.2)$ \\
\hline Yes & $47(16.8)$ \\
\hline \multicolumn{2}{|l|}{ Alcohol use } \\
\hline No & $208(74.6)$ \\
\hline Yes & $71(25.4)$ \\
\hline \multicolumn{2}{|l|}{ Exercise } \\
\hline No & $52(18.6)$ \\
\hline Yes & $227(81.4)$ \\
\hline CRSOM-31 symptom-impact score & $175.4 \pm 104.3(158.0,2-472)^{*}$ \\
\hline$<116$ & $94(33.7)$ \\
\hline $117-205$ & $92(33.0)$ \\
\hline$>205$ & $93(33.3)$ \\
\hline CRSOM-31 nasal symptoms score & $42.0 \pm 24.0(39.0,0-108)$ \\
\hline CRSOM-31 eye symptoms score & $9.1 \pm 10.2(5.0,0-40)$ \\
\hline CRSOM-31 sleep score & $21.9 \pm 18.2(19.0,0-60)$ \\
\hline CRSOM-31 ear symptoms score & $15.8 \pm 16.2(12.0,0-66)$ \\
\hline CRSOM-31 general symptoms score & $35.5 \pm 29.5(28.0,0-135)$ \\
\hline CRSOM-31 practical problems score & $29.4 \pm 20.4(25.0,0-91)$ \\
\hline CRSOM-31 emotional consequences score & $21.8 \pm 17.8(20.0,0-60)$ \\
\hline \multicolumn{2}{|l|}{ Etiology of rhinitis } \\
\hline \multicolumn{2}{|l|}{ Allergic rhinitis } \\
\hline No & $132(47.3)$ \\
\hline Yes & $147(52.7)$ \\
\hline
\end{tabular}


TABle 1: Continued.

\begin{tabular}{lc}
\hline Characteristic & $n(\%)$ \\
\hline Deviation of nasal septum & $234(83.9)$ \\
No & $45(16.1)$ \\
Yes & $194(69.5)$ \\
Chronic hypertrophic rhinitis & $85(30.5)$ \\
No & \\
Yes & $127(45.5)$ \\
Chronic rhinosinusitis & $50(17.9)$ \\
No & $102(36.6)$ \\
Without nasal polyps & \\
With nasal polyps & \\
\hline
\end{tabular}

${ }^{*}$ Mean \pm standard deviation (median, minimum-maximum).

topical medications). Fair or poor general health status was significantly associated with the use of pharmacology treatment modality and especially with the use of topical medications, whereas very good or excellent general health status was inversely associated with the use of surgical intervention. Smoking was significantly associated with the use of nasal irrigation. Alcohol use was significantly associated with the pharmacology treatment modality. Worse diseasespecific quality of life (CRSOM-31 symptom-impact score) was significantly associated with the use of pharmacology treatment modality, oral medications, and traditional Chinese medicine. In terms of the etiologies of rhinitis, allergic rhinitis was significantly associated with topical medications. Deviation of nasal septum was significantly associated with surgical intervention. Finally, chronic rhinosinusitis, both with or without nasal polyps, was significantly associated with the use of topical medications, surgical intervention, and the use of nasal irrigation.

\section{Discussion}

Rhinitis and rhinosinusitis are common health problems that impact on the quality of life of their sufferers [11, 12]. They also impose a substantial burden on the healthcare resources [13]. The World Health Organization proposed a stepwise treatment for allergic rhinitis in the Allergic Rhinitis and its Impact on Asthma (ARIA) guideline [2]. Oral antihistamine and topical corticosteroid are recommended for the first-line treatment for mild and moderate-severe allergic rhinitis by the ARIA guideline. Our results showed that most rhinitis patients had received pharmacological treatments (85.7\%) which included the uses of oral medications $(77.1 \%)$ and topical medications (65.9\%), indicating that guideline-directed rhinitis treatment is widely adopted by both physicians and patients in Taiwan. In addition, our data showed that approximately a third of the patients (34.1\%) had used nasal irrigation to relieve their rhinitis symptoms. Nasal irrigation is used for various sinonasal condition and postoperative care after nasal surgery [14-16]. The mechanisms of nasal irrigation include physical cleaning, enhancement of mucociliary function, and removal of local inflammatory mediators. Although it is widely recommended and commonly used in Taiwanese patients, there was no data reporting the proportion of nasal irrigation use in clinical settings.

About one third of our patients (34.4\%) had used TCM for their rhinitis symptoms. The relatively common use of it could partly be explained by the health care system of Taiwan because TCM services are fully reimbursed by the National Health Insurance program [17]. Based on the results of an analysis of TCM outpatient reimbursement claims from 1996 to 2001 in Taiwan, diseases of the respiratory system were found to rank at the top of the major disease categories for TCM visits, which accounted for $27 \%$ of all TCM visits [18].

Understanding the factors associated with the use of different treatment modalities for rhinitis can be helpful for clinicians when they are considering the choice of treatment for their patients. Our results demonstrated that sex, educational levels, general health status, smoking habit, alcohol use, and disease-specific quality of life were independent factors associated with different treatment modalities. These associations had been adjusted for the etiologies of rhinitis and remained statistically significant. In particular, male patients were more likely to receive surgical interventions, while lower educational levels were associated with the use of oral and topical medications.

Patients with poor or fair general health status were associated with the use of topical medications. A possible reason is that the occurrence of drug-to-drug interactions is relatively rare with topical medications and therefore, suitable for patients who were on medications for their other diseases. It is of interest to note that very good or excellent general health status was inversely associated with surgical intervention. Since surgery for rhinitis is typically performed under local anesthesia, the general health status of a patient should not be a contraindication for surgery. The reasons for the association between the use of surgical intervention and general health status will require further investigations.

Nasal irrigation is a method to relieve sinus symptoms by rinsing the nasal cavity with isotonic or hypertonic saline. A Cochrane systematic review on eight randomized controlled trials concluded that the use of topical saline could be 


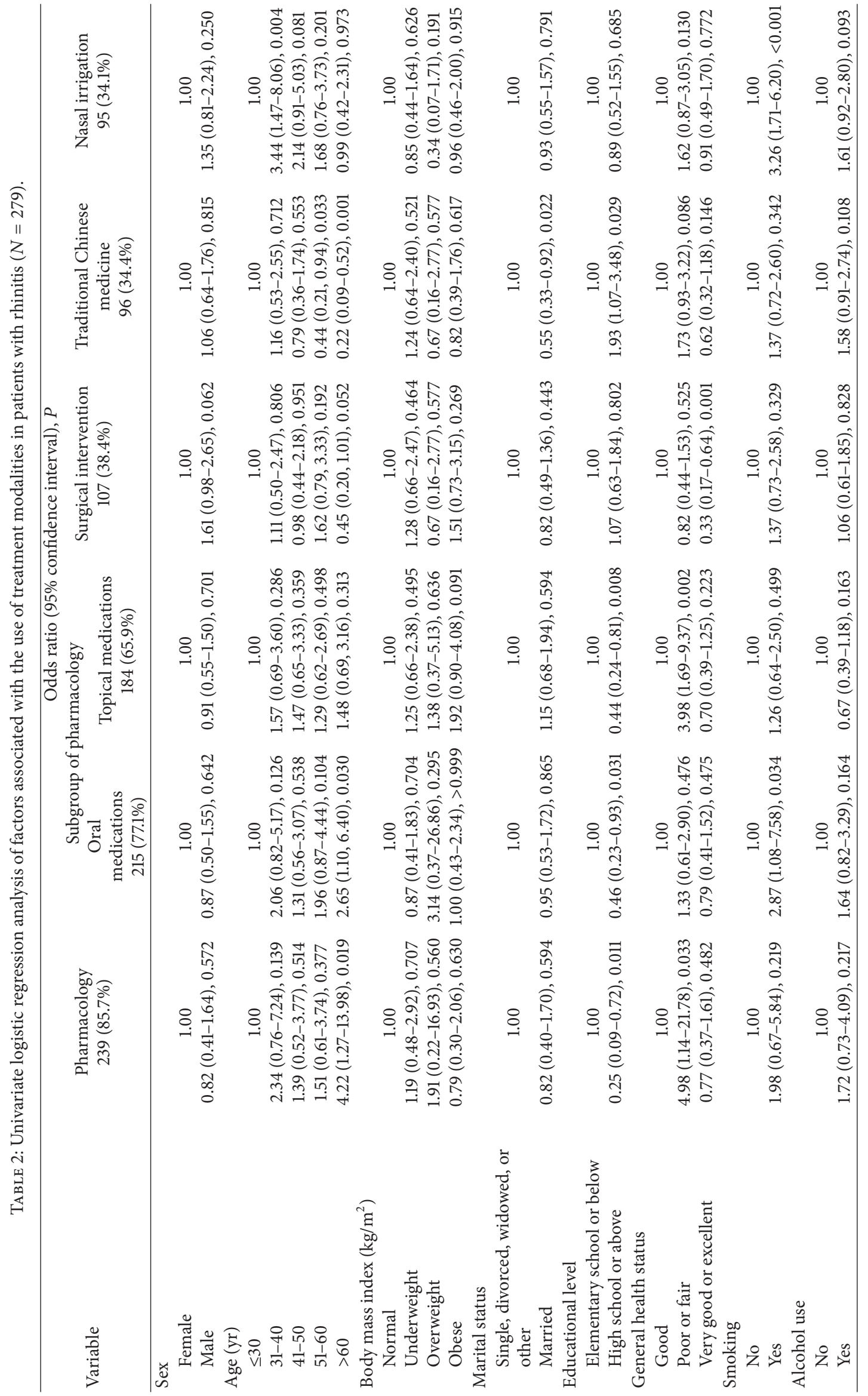




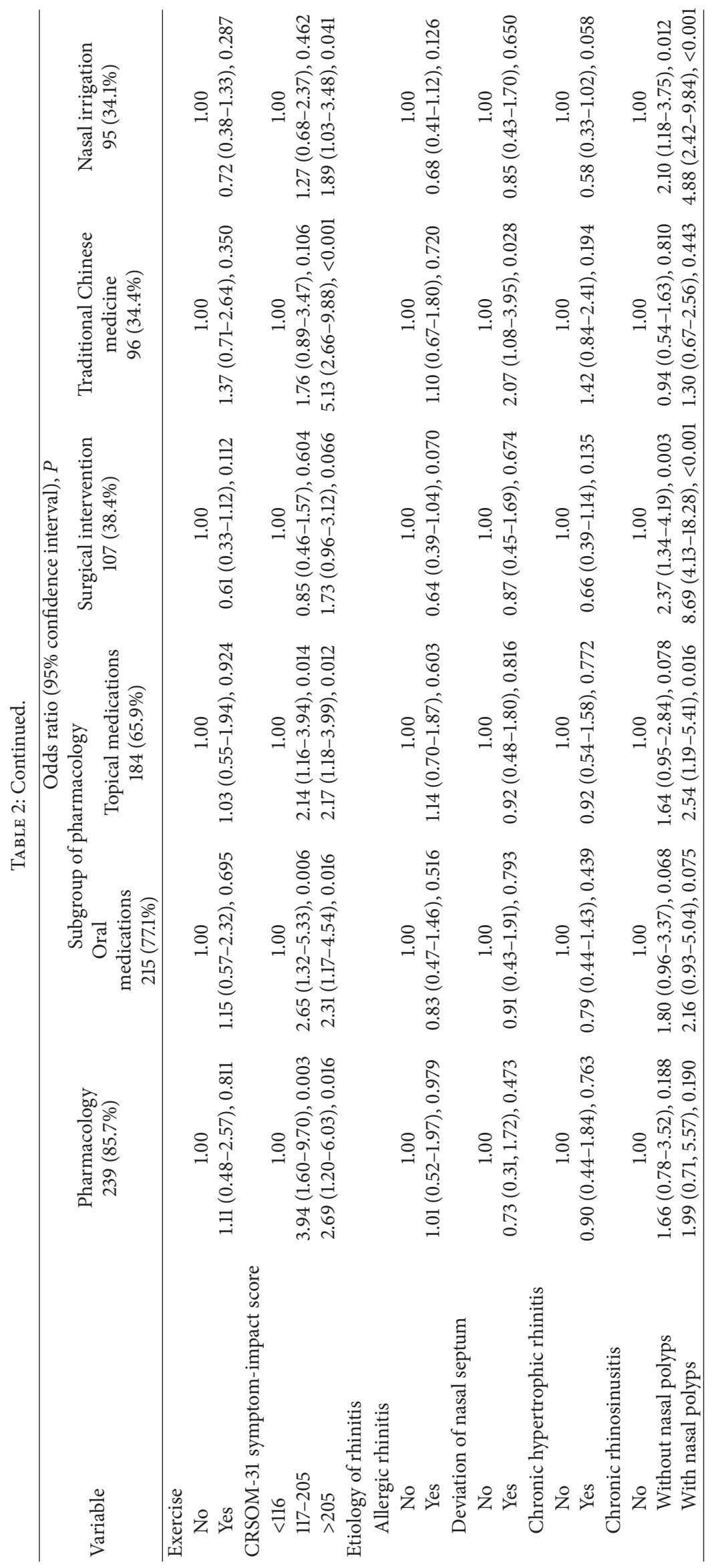




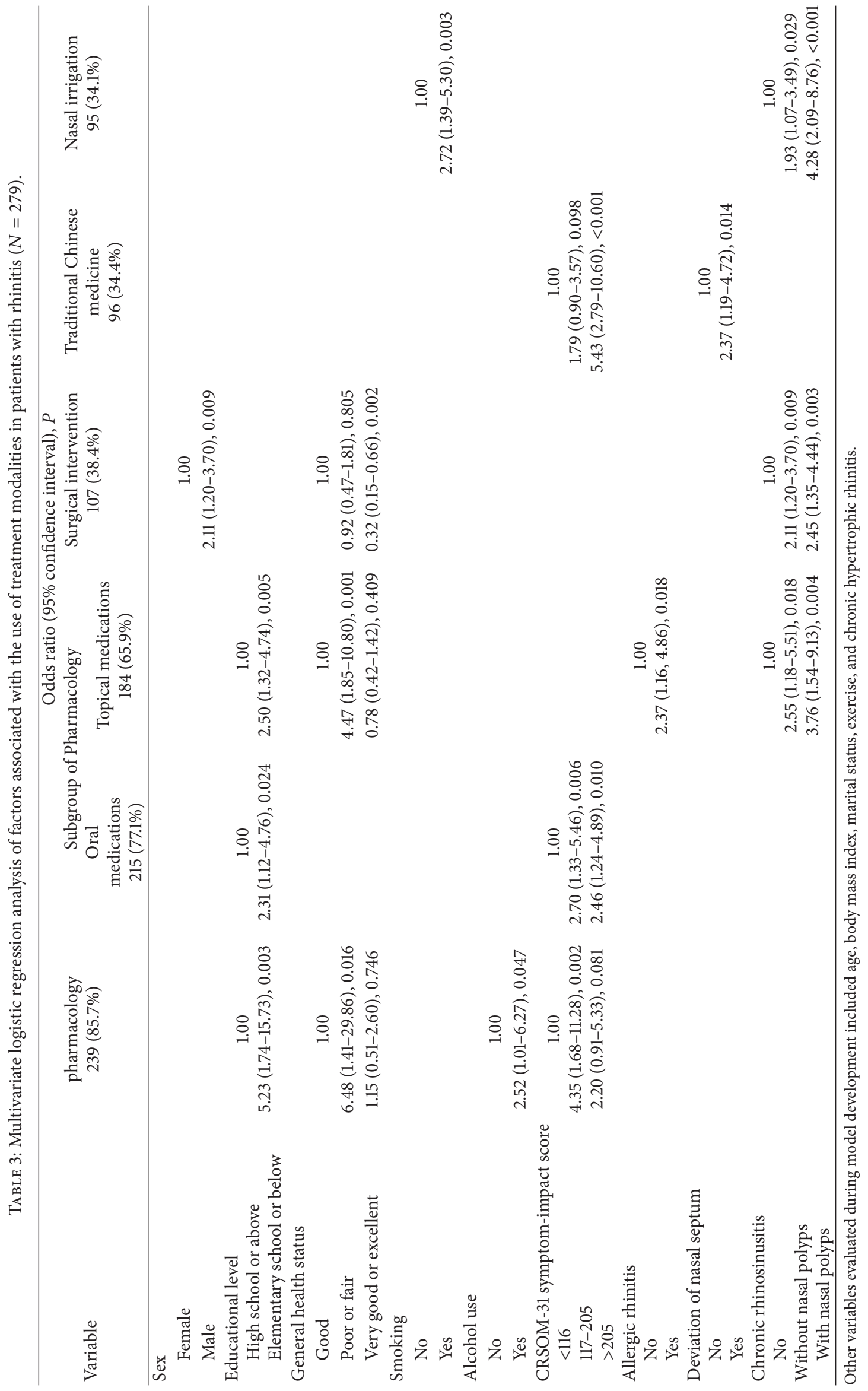


included as a treatment adjunct for the symptoms of chronic rhinosinusitis [19]. In our study, smoking was significantly associated with the use of nasal irrigation. Nasal irrigation has been claimed as a method to remove nicotine and tar buildup in the nasal passages for smokers, and this might explain the association between nasal irrigation and smoking observed in this study. Previous large-scale population studies have revealed that cigarette smoking and chronic rhinitis were associated in a dose-dependent manner [20]. In addition, past and current secondhand tobacco smoke exposure was reported to be a risk factor for allergic rhinitis. Subjects with secondhand tobacco smoke exposure and allergic rhinitis were significantly more likely to use nasal decongestants [21]. However, no studies have specifically investigated whether smoking was associated with increased use of nasal irrigation.

Finally, worse disease-specific quality of life, assessed by the use of CRSOM-31, was found to be associated with the use of oral medications and TCM. Since it is likely that the chronicity of rhinitis symptoms is associated with a worse quality of life, patients with worse quality of life might seek alternative therapies including TCM as a way to minimize the adverse effects of chronic use of conventional medications [22]. This might explain the association between CRSOM-31 and the use of TCM. The association between CRSOM-31 and the use of oral medications could be explained by the fact that oral medication is often used for moderate or severe cases not responsive to topical medications, which are likely to be cases with worse quality of life.

This study has some limitations that need to be considered. First, we did not directly measure the severity of rhinitis in our patients. It is possible that the choice of treatment modalities was affected by disease severity. Nevertheless, we have included CRSOM-31 symptom-impact scores in our multivariate analyses as a proxy to control for the effect of severity of rhinitis. Second, all patients were recruited from one medical center, and most of them had previously been treated for rhinitis by their primary care physicians. Therefore, they might represent cases of greater severity.

\section{Conclusions}

Results from the present study indicated that common treatment modalities in Taiwanese patients with rhinitis included oral medications, topical medications, surgical intervention, TCM, and nasal irrigation. The use of different treatment modalities among Taiwanese patients with rhinitis was associated with different factors including sex, educational levels, general health status, smoking habit, alcohol use, and diseasespecific quality of life, adjusting for etiologies of rhinitis.

\section{List of Abbreviations}

CRSOM-31: Chinese version of the 31-item rhinosinusitis outcome measure

TCM: $\quad$ Traditional Chinese medicine.

\section{Conflict of Interests}

The authors declare that they have no conflict of interests.

\section{Authors' Contribution}

Kai-Li Liang, Rong-San Jiang, and Yueh-Chiao Yeh conceived the research questions. Kai-Li Liang, Yueh-Chiao Yeh, and Malcolm Koo designed the study. Hsin Tsao interviewed and enrolled the participants. Hsin Tsao and Yueh-Chiao Yeh undertook the data analyses. Ting-Ting Yen and Kai-Li Liang helped to draft the paper. Malcolm Koo and Yueh-Chiao Yeh made critical revision of the paper for important intellectual content. All authors read and approved the final paper.

\section{References}

[1] D. V. Wallace, M. S. Dykewicz, D. I. Bernstein et al., "The diagnosis and management of rhinitis: an updated practice parameter," The Journal of Allergy and Clinical Immunology, vol. 122, no. 2, supplement, pp. S1-S84, 2008.

[2] J. L. Broek, J. Bousquet, C. E. Baena-Cagnani et al., "Allergic rhinitis and its impact on asthma (ARIA) guidelines: 2010 revision," The Journal of Allergy and Clinical Immunology, vol. 126, no. 3, pp. 466-476, 2010.

[3] W. W. Carr, M. R. Nelson, and J. A. Hadley, "Managing rhinitis: strategies for improved patient outcomes," Allergy and Asthma Proceedings, vol. 29, no. 4, pp. 349-357, 2008.

[4] Y. G. Hipps, Y. E. Hacker, D. L. Hoffmann, J. A. Brinckmann, R. R. Socci, and D. Rogers, "Self-reported quality of life in complementary and alternative medicine treatment of chronic rhinosinusitis among African Americans: a preliminary, openlabel pilot study," Journal of Alternative and Complementary Medicine, vol. 15, no. 1, pp. 67-77, 2009.

[5] Y. Y. Kung, Y. C. Chen, S. J. Hwang, T. J. Chen, and F. P. Chen, "The prescriptions frequencies and patterns of Chinese herbal medicine for allergic rhinitis in Taiwan," Allergy, vol. 61, no. 11, pp. 1316-1318, 2006.

[6] S. S. Mukerji, M. A. Pynnonen, H. M. Kim, A. Singer, M. Tabor, and J. E. Terrell, "Probiotics as adjunctive treatment for chronic rhinosinusitis: a randomized controlled trial," OtolaryngologyHead and Neck Surgery, vol. 140, no. 2, pp. 202-208, 2009.

[7] M. G. Stewart, "Evidence-based medicine in rhinology," Current Opinion in Otolaryngology \& Head \& Neck Surgery, vol. 16, no. 1, pp. 14-17, 2008.

[8] W. J. Fokkens, V. J. Lund, and J. Mullol, “EPOS 2012: European position paper on rhinosinusitis and nasal polyps 2012. A summary for otorhinolaryngologists," Rhinology, vol. 50, no. 1, pp. 1-12, 2012.

[9] K. L. Liang, T. K. Lin, H. S. Hao et al., "Validation of the chinese version of the 31-item rhinosinusitis outcome measure," Journal of Taiwan Otolaryngology-Head \& Neck Surgery, vol. 41, no. 4, pp. 121-128, 2006.

[10] J. F. Piccirillo, D. Edwards, A. Haiduk, C. Yonan, and S. E. Thawley, "Psychometric and clinimetric validity of the 31-item rhinosinusitis outcome measure (RSOM-31)," American Journal of Rhinology, vol. 9, no. 6, pp. 297-306, 1995.

[11] V. J. Lund, "Impact of chronic rhinosinusitis on quality of life and health care expenditure," Clinical allergy and immunology, vol. 20, pp. 15-24, 2007. 
[12] T. Ozdoganoglu, M. Songu, and H. M. Inancli, "Quality of life in allergic rhinitis," Therapeutic Advances in Respiratory Disease, vol. 6, no. 1, pp. 25-39, 2012.

[13] G. W. Canonica, J. Bousquet, J. Mullol, G. K. Scadding, and J. C. Virchow, "A survey of the burden of allergic rhinitis in Europe," Allergy, vol. 62, no. supplement 85, pp. 17-25, 2007.

[14] K. E. Hermelingmeier, R. K. Weber, M. Hellmich, C. P. Heubach, and R. Mösges, "Nasal irrigation as an adjunctive treatment in allergic rhinitis: a systematic review and meta-analysis," American Journal of Rhinology \& Allergy, vol. 26, no. 5, pp. el19e125, 2012.

[15] K. L. Liang, M. C. Su, H. C. Tseng, and R. S. Jiang, "Impact of pulsatile nasal irrigation on the prognosis of functional endoscopic sinus surgery," Journal of Otolaryngology-Head and Neck Surgery, vol. 37, no. 2, pp. 148-153, 2008.

[16] R. Harvey, S. A. Hannan, L. Badia, and G. Scadding, "Nasal saline irrigations for the symptoms of chronic rhinosinusitis," Cochrane Database of Systematic Reviews, no. 3, Article ID CD006394, 2007.

[17] D. Wu, "The healthcare system in Taiwan," World Hospitals and Health Services, vol. 42, no. 1, pp. 14-17, 2006.

[18] F. P. Chen, T. J. Chen, Y. Y. Kung et al., "Use frequency of traditional Chinese medicine in Taiwan," BMC Health Services Research, vol. 7, article 26, 2007.

[19] R. Harvey, S. A. Hannan, L. Badia, and G. Scadding, "Nasal saline irrigations for the symptoms of chronic rhinosinusitis," Cochrane Database of Systematic Reviews, no. 3, Article ID CD006394, 2007.

[20] J. Eriksson, L. Ekerljung, B. M. Sundblad et al., "Cigarette smoking is associated with high prevalence of chronic rhinitis and low prevalence of allergic rhinitis in men," Allergy, vol. 68, no. 3, pp. 347-354, 2013.

[21] S. Y. Lin, D. D. Reh, S. Clipp, L. Irani, and A. Navas-Acien, "Allergic rhinitis and secondhand tobacco smoke: a populationbased study," American Journal of Rhinology and Allergy, vol. 25, no. 2, pp. e66-e71, 2011.

[22] H. Guo and M. P. Liu, "Mechanism of traditional Chinese medicine in the treatment of allergic rhinitis," Chinese Medical Journal, vol. 126, no. 4, pp. 756-760, 2013. 


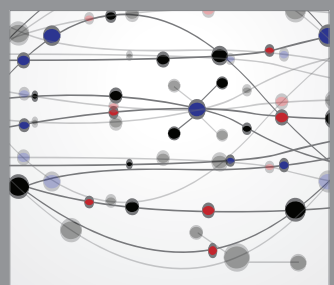

The Scientific World Journal
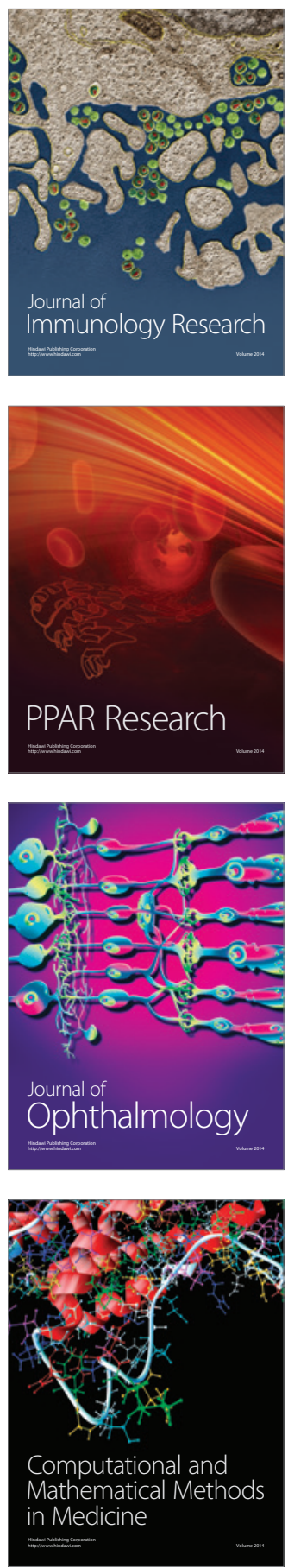

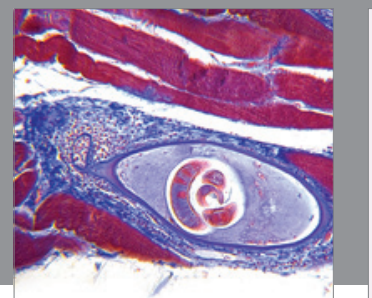

Gastroenterology

Research and Practice
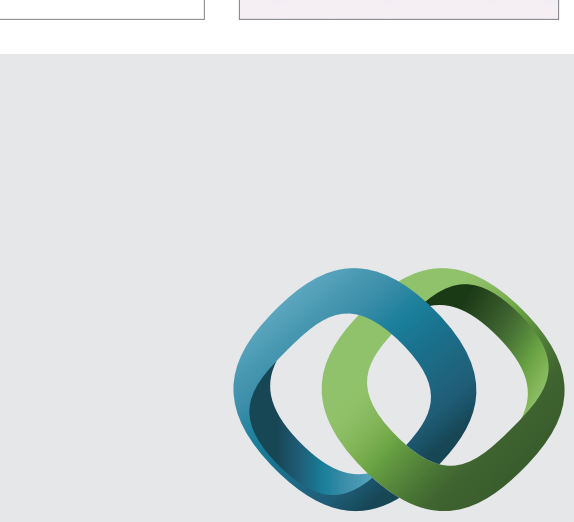

\section{Hindawi}

Submit your manuscripts at

http://www.hindawi.com
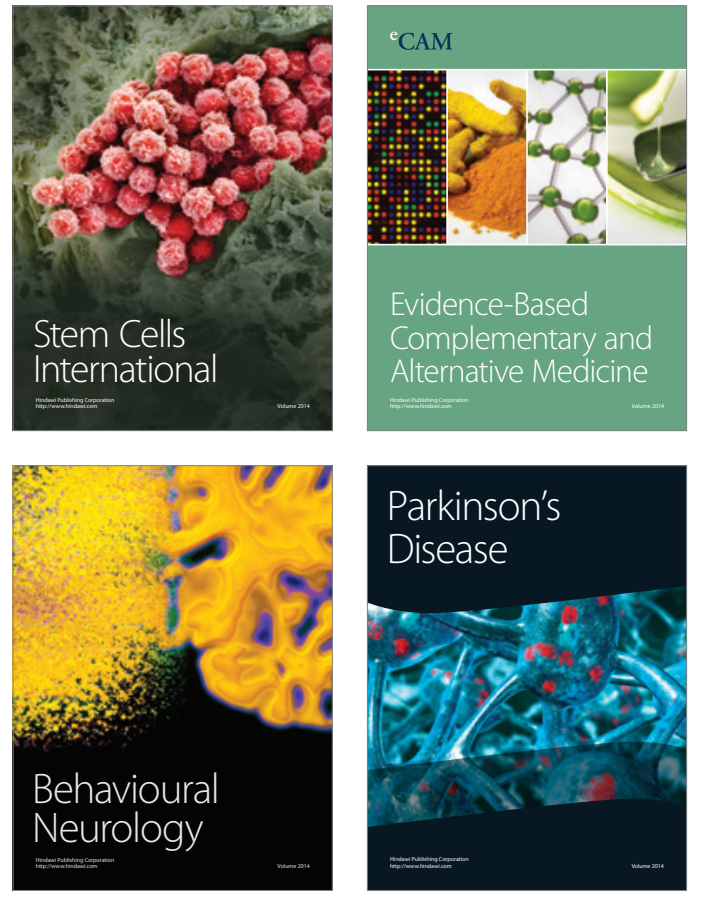
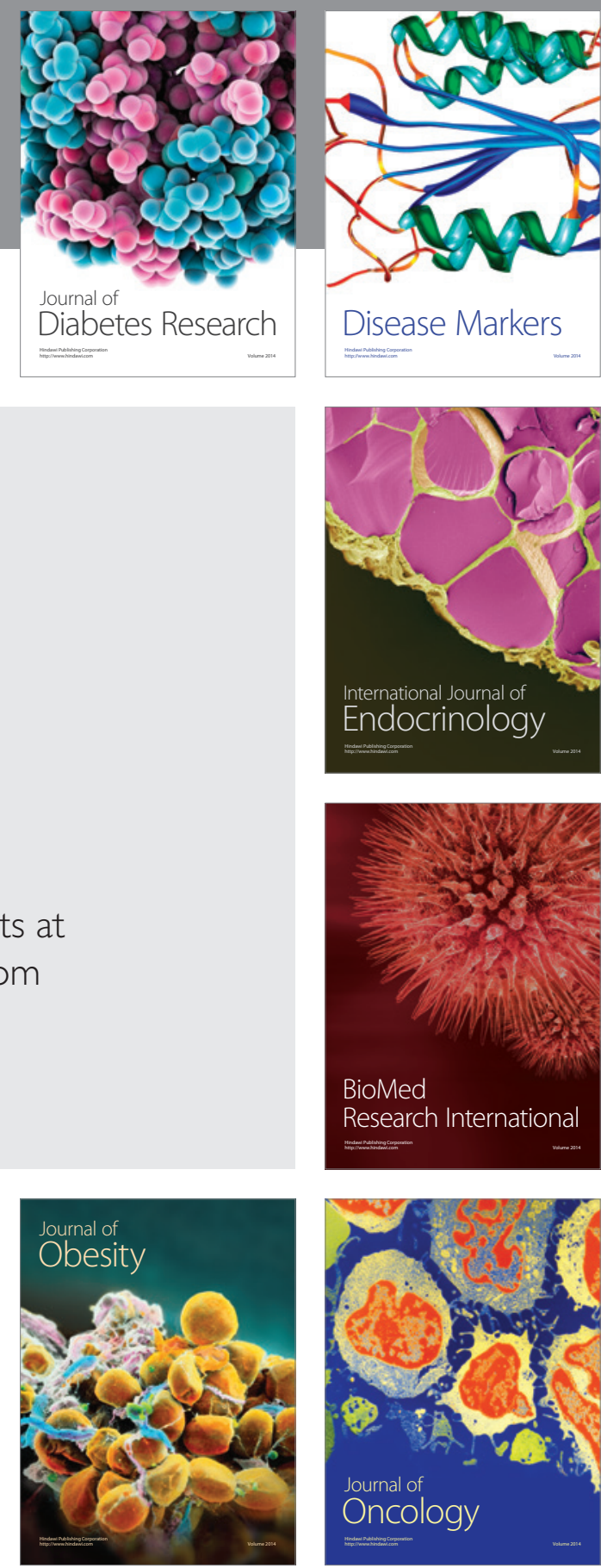

Disease Markers
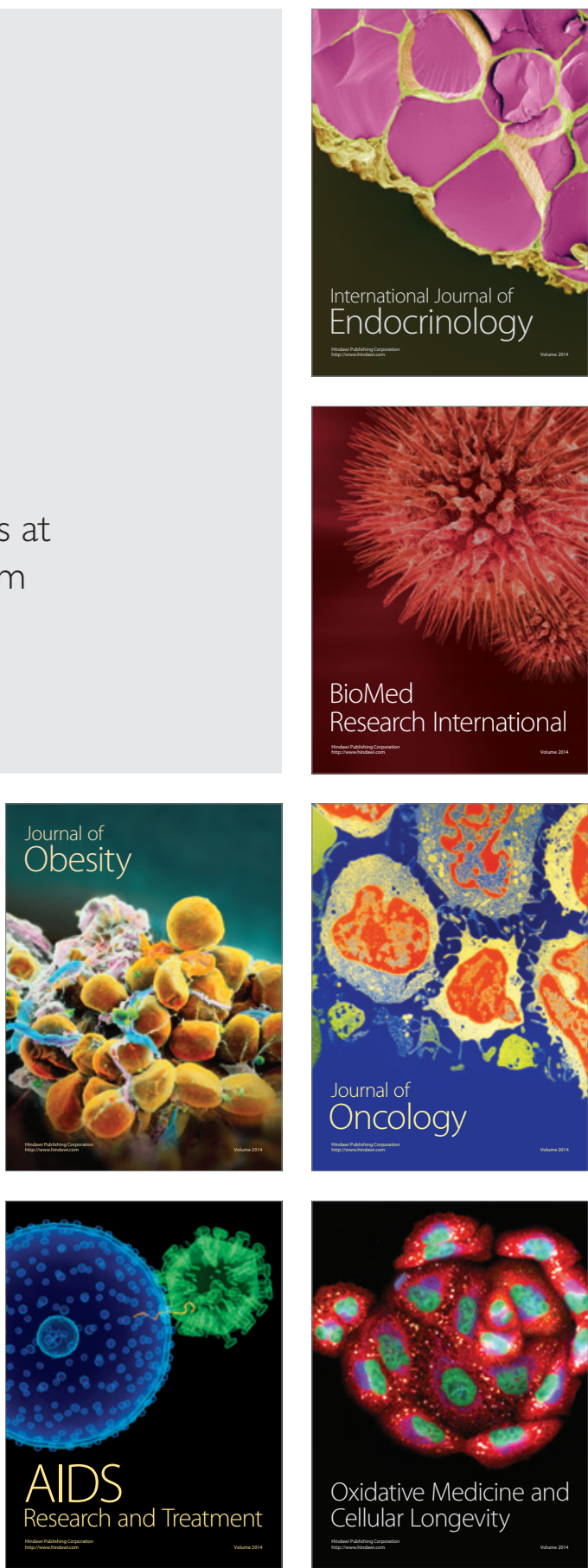